\title{
Novel Synthesis of Chiral 5-Cyanomethyl-3,4-dihydroxy-2-iodomethyltetrahydrofuran from 3-Diphenylmethyl-5-(1,2-dihydroxy-3-butenyl)isoxazolines via Iodoetheration Reaction
}

\author{
Min Jung Seo, Bum Suk Son, Bo Gan Song. Bok Ju Song. Sang Dae Lee. \\ Ji Young Kim, Hak Do Kim, Zaesung No, and Hyoung Rae Kim* \\ Medicinal Science Division, Korea Research Institute of Chemical Technology, P. O. Box 107, Yuseong, Daejeon 305-600, Korea \\ "E-mail: hyungrk@krist.re.kr \\ Received Augtst 2, 2005
}

Key Words : 5-Cyanomethyl-3,4-dihydroxy-2-iodomethyltetrahydroturan, 1,3-Dipolar cycloaddition, Isoxazoline, Iodoetheration, Diastereoselectivity

3-Butenyl-isoxazolines were known as good precursors for the syntheses of new types of 5-cyanomethyl-2jodomethyltetrahydrofuran derivatives. 5-Cyanomethyl-2jodomethyltetrahydrofuran was synthesized from isoxazolines by electrophilic jodoetheration using jodine or jodine monochloride, ${ }^{1}$ which was the first example of electrophilic cleavage of isoxazoline ring by iodoetheration reaction. From the combination of this reaction and diastereoselective formation of isoxazoline in the support of magnesium chelation effect, ${ }^{2}$ we synthesized 5-cyanomethyl-3-hydroxy2-iodomethyltetrahydrofuran from syn-5-(1-hydroxy-3butenyl)isoxazolines with diastereoselctivity. ${ }^{3}$ We expected better diastereoselectivity could be achieved by adding one more hydroxyl group at the 2-position of butenyl group in isoxazoline close to the reaction center: First, we prepared chiral 3-diphenylmethyl-5-\{1,2-dihydroxy-3-butenyl)isoxazolines $(3)^{4}$ by 1,3-dipolar cycloaddition reaction of diphenylacetohydroximoyl chloride (1) with $(3 R .4 R)-1,5-$ hexadiene-3,4-diol ${ }^{5}$ (2a) and meso-1,5-hexadiene-3,4-diol ${ }^{6}$ (2b) by the aid of magnesium chelation effect, and after $O$ protection of isoxazolines, iodoetheration was examined to synthesize chiral 5-cyanomethyl-3,4-dihydroxy-2-iodomethyltetrahydrofurans 6.
Diphenylacetohydroximoyl chloride (1), precursor of diphenylacetonitrile oxide was reacted with $(3 R, 4 R)-1,5-$ hexadiene-3,4-diol (2a) in the presence of ethylmagnesium bromide as shown in Scheme 1. The more ethylmagnesium bromide was used, the more diadduct $\mathbf{4 a}^{7}$ was formed in this reaction. When 2.2 equiv of ethylmagnesium bromide was used, the best result of 3a was obtained (Entry 3 in Scheme 1). In case of meso-1,5-hexadiene-3,4-diol (2b), however, only $47 \%$ of 3 b was obtained (Entry 4 in Scheme 1). The isolated 3 a was a single stereoisomer that thought to be synisomer of 5-position and a-position due to the magnesium chelation effect, but $\mathbf{3 b}$ must be a racemic mixture of $s y n-$ isomers according to which double bond was reacted.

The hydroxyl groups of 3a were protected with TBDMS and MOM group, and then reacted with jodine monochloride in dichloromethane to give 5-cyanomethyl-3,4dihydroxy-2-iodomethyltetrahydrofurans $\mathbf{6}$ in moderate yields as shown in Scheme 2. 5a ( $\mathrm{P}=\mathrm{TBDMS}$ ) afforded only trons-6a (P=TBDMS) without any trace of cis-6a ( $\mathrm{P}=$ TBDMS), while 5a ( $\mathrm{P}=\mathrm{MOM}$ ) afforded a mixture of two isomers (trans/cis, 5/1). As we expected, the bulkier TBDMS group showed better diastereoselectivity in iodoetheration. We examined the energy minimizations of trans-

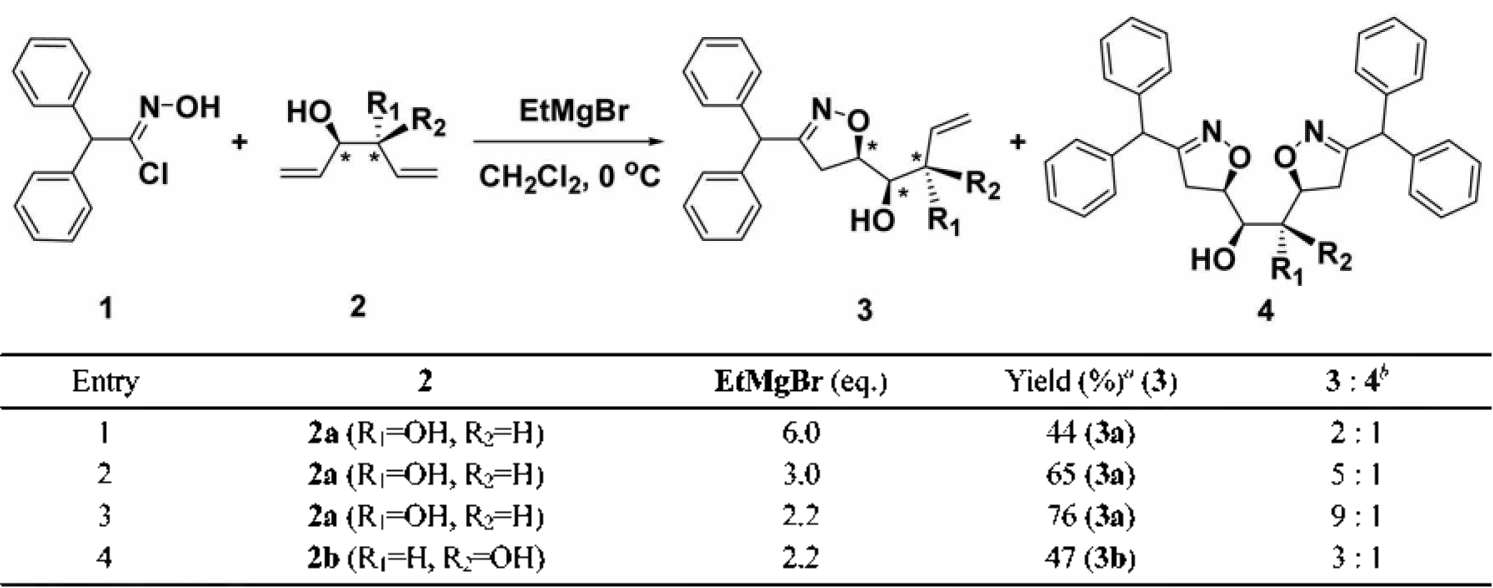

TSolated yields. 'Tsolated ratio. 


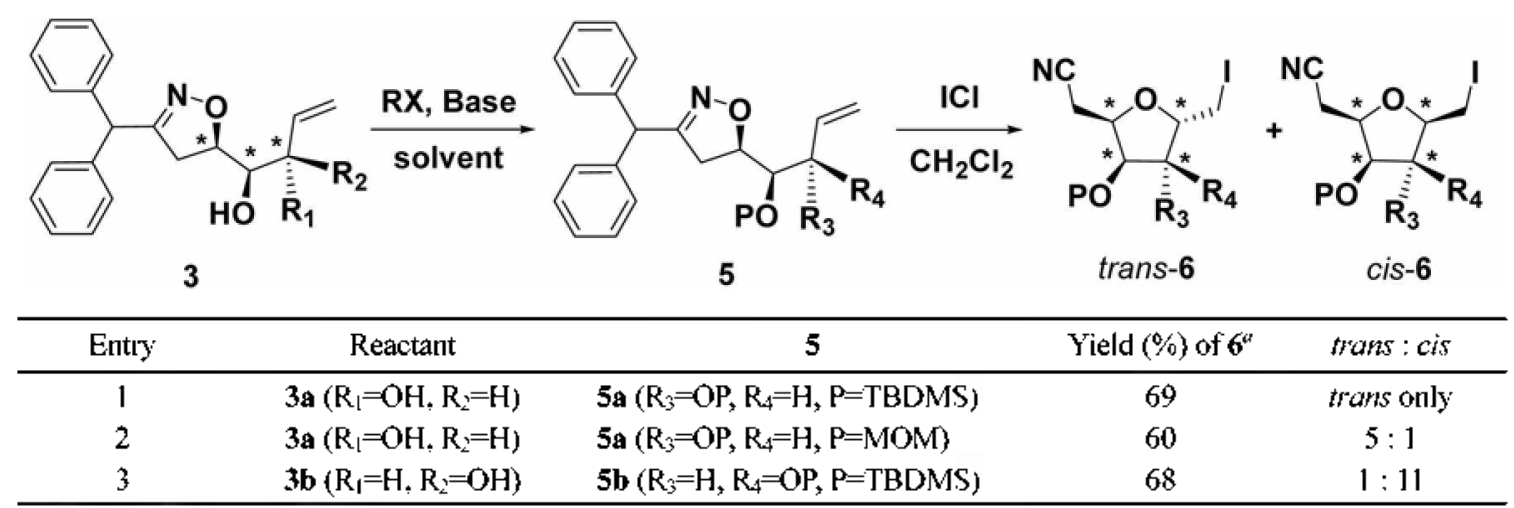

${ }^{\star}$ Tsolated yields ( trans-6 + cis-6).

Scheme 2

6a and cis-6a with other protection group by MM2. TBDMS-Protected 6a showed about $1 \mathrm{kcal}$ difference between $\operatorname{trans}$-6a and cis-6a, and MOM-protected $6 \mathrm{a}$ showed almost no difference between two isomers. Interestingly, 3b derived from meso-1,5-hexadiene-3,4-diol (2b) was protected with TBDMS group and the iodoetheration of $\mathbf{5 b}$ ( $\mathrm{P}=\mathrm{TBDMS}$ ) with iodine monochloride afforded $\mathrm{I}: 1 \mathrm{I}$ mixture of trans-6b ( $\mathrm{P}=$ TBDMS) and cis $-6 \mathrm{~b}(\mathrm{P}=\mathrm{TBDMS})$. In the MM2 calculation, cis-6b $(\mathrm{P}=\mathrm{TBDMS})$ was more stable by $1.6 \mathrm{kcal}$ than trans $-6 \mathrm{~b}$ ( $\mathrm{P}=\mathrm{TBDMS})$. The individual five isomers of 6 such as trans-6a ( $\mathrm{P}=\mathrm{TBDMS}$ ), trans-6a ( $\mathrm{P}=\mathrm{MOM}$ ), cis-6a ( $\mathrm{P}=\mathrm{MOM})$, trans-6b ( $\mathrm{P}=\mathrm{TBDMS})$, and cis-6b ( $\mathrm{P}=\mathrm{TBDMS})$ were separated, and the assignments of each proton and the relative stereochemistry of each isomer were confirmed by COSY and NOE experiment. For example, when methylene protons attached to jodine in trans $-6 \mathrm{~b}(\mathrm{P}=\mathrm{TBDMS})$ appeared at $3.22 \mathrm{ppm}$ was irradiated, protons on 4,5-positions (3.93-4.04 ppm) and 2,3positions (4.28-4.34 ppm) showed $9.52 \%$ and $2.13 \%$ NOE respectively. However, when methylene protons attached to jodine in cis-6b ( $\mathrm{P}=$ TBDMS) appeared at 3.28 ppm was irradiated, only protons on 4,5-positions (4.17-4.23 ppm) showed $5.45 \%$ NOE with $0.75 \%$ NOE of methylene attached to $\mathrm{CN}$ group. The weak NOE between two methylene groups was thought to be a critical evidence of $\mathrm{cis}$-form. The configurations of other tetrahydrofurans 6 were confirmed by the same experiments.

As a conclusion, 3-diphenylmethyl-5-(1,2-dihydroxy-3butenyl)isoxazoline was prepared diastereoselectively by 1,3-dipolar cycloaddition reaction of diphenylacetonitrile oxide with chiral 1,5-hexadiene-3,4-diol in the support of magnesium chelation effect.

Iodoetheration of $O$-protected 3-diphenylmethyl-5-(1,2dihydroxy-3-butenyl)isoxazolines afforded the corresponding 5-cyanomethyl-3,4-di( $O$-protected-hydroxy)-2-jodomethyltetrahydrofurans with good diastereoselectivity. From these reactions, new series of highly substituted chiral tetrahydrofurans could be synthesized.

\section{Experimental Section}

General. Unless otherwise specified, all reagents were purchased from commercial sources and were used without further purification. 'H NMR spectra were obtained with a Bruker AMX-500. All chemical shifts are reported in ppm downfield from intemal tetramethylsilane and coupling constants are given in $\mathrm{Hz}$. IR spectra were taken by a Sense ATR FT-IR spectrophotometer. MS spectra were recorded by EI method and HRMS spectra were measured on a Jeol JMX-DX 303 mass spectrometer. Chromatographic separations were carried out on a silica gel column (Merck silica gel 60).

Typical procedure of 1,3-dipolar cycloaddition (Entry 3 in Scheme 1): To a solution of $(3 R, 4 R)-1,5$-hexadiene-3,4diol (2a, $114 \mathrm{mg}, 1.0 \mathrm{mmol})$ in dichloromethane $(20 \mathrm{~mL})$ was added ethylmagnesium bromide $(3.0 \mathrm{M}$ solution in diethyl ether, $0.74 \mathrm{~mL}, 2.2 \mathrm{mmol}$ ) at $0^{\circ} \mathrm{C}$ and the reaction mixture was stirred for $30 \mathrm{~min}$ at this temperature. Diphenylacetohydroximoyl chloride $(246 \mathrm{mg}, 1.0 \mathrm{mmol})$ in dichloromethane $(5 \mathrm{~mL})$ was added slowly by a syringe at 0 ${ }^{\circ} \mathrm{C}$. The reaction mixture was stirred for $2 \mathrm{~h}$ at $\mathrm{rt}$ and saturated ammonium chloride solution $(10 \mathrm{~mL}$ ) was poured into the reaction mixture. The organic layer was extracted with dichloromethane $(50 \mathrm{~mL})$, washed with brine, dried over $\mathrm{MgSO}_{4}$, and concentrated by a rotary evaporator. The residue was separated with silica gel chromatography ( $n$ hexane/ethyl acetate, $2 / 1)$ to give $3 a(246 \mathrm{mg}, 76 \%)$ as a white solid.

3a: 'H NMR ( $\left.500 \mathrm{MHz}^{\mathrm{CODCl}}\right) \delta 2.48(\mathrm{~d}, \mathrm{IH},-\mathrm{OH}, J=$ $7.7 \mathrm{~Hz}), 2.61(\mathrm{~d}, \mathrm{H},-\mathrm{OH}, J=3.4 \mathrm{~Hz}), 2.98(\mathrm{dd}, \mathrm{IH}, J=10.7$, $16.9 \mathrm{~Hz}$ ), 3.02 (dd, $1 \mathrm{H}, J=8.1,16.9 \mathrm{~Hz}), 3.38-3.42(\mathrm{~m}, \mathrm{IH})$, 4.21-4.24 (m, IH), 4.71 (ddd, $1 \mathrm{H}, J=10.7,8.1,2.9 \mathrm{~Hz}$ ), $5.15(\mathrm{~s}, 1 \mathrm{H}), 5.23-5.26(\mathrm{~m}, 2 \mathrm{H}), 5.81-5.94(\mathrm{~m}, 1 \mathrm{H}), 7.22-$ $7.43(\mathrm{~m}, 10 \mathrm{H}) ;{ }^{13} \mathrm{C} \mathrm{NMR}\left(\mathrm{CDCl}_{3}, 125 \mathrm{MHz}\right) \delta 39.07,50.33$, $73.81,74.92,80.68,117.87,127.25,127.31,128.63,128.70$, $128.71,128.83,136.51,139.35,160.96$; IR (neat) 3399 , $3028,2921,1599,1494,1452,1324,1079,994,905 \mathrm{~cm}^{-1}$; HRMS calcd for $\mathrm{C}_{20} \mathrm{H}_{2} \mathrm{NO}_{3} 323.152144$, found 323.151957 .

3b: 'H NMR $\left(500 \mathrm{MHz}, \mathrm{CDCl}_{3}\right) \delta 2.5 \mathrm{I}(\mathrm{d}, \mathrm{IH},-\mathrm{OH}, J=$ $7.7 \mathrm{~Hz}), 2.61(\mathrm{~d}, \mathrm{H},-\mathrm{OH}, J=8.1 \mathrm{~Hz}), 2.96(\mathrm{dd}, \mathrm{IH}, J=10.5$, $16.9 \mathrm{~Hz}), 3.05$ (dd, $1 \mathrm{H}, J=8.5,16.9 \mathrm{~Hz}), 3.44-3.53(\mathrm{~m}, \mathrm{IH})$, $4.21-4.31$ (m, IH), 4.82 (ddd, $1 \mathrm{H}, J=10.5,8.5,2.4 \mathrm{~Hz}$ ), $5.16(\mathrm{~s}, 1 \mathrm{H}), 5.24-5.42(\mathrm{~m}, 2 \mathrm{H}), 5.85-6.02(\mathrm{~m}, 1 \mathrm{H}), 7.22-$ $7.38(\mathrm{~m}, 10 \mathrm{H}) ;{ }^{13} \mathrm{C} \mathrm{NMR}\left(\mathrm{CDCl}_{3}, 125 \mathrm{MHz}\right) \delta 39.24,50.35$, 
$74.24,74.35,79.90,117.05,127.25,127.30,128.63,128.70$, $128.76,128.81,136.74,139.34,139.36,161.15$; IR (neat) $3410,3028,2923,1494,1452,1320,1080,998 \mathrm{~cm}^{-1}$; HRMS caled for $\mathrm{C}_{20} \mathrm{H}_{21} \mathrm{NO}_{3} 323.152144$, found 323.151871 .

Protection of hydroxyl group: The protection of 3 with TBDMS or MOM group was carried out in good yields by general procedure.

5a $(\mathrm{P}=\mathrm{TBDMS})$ : 'H NMR $\left(500 \mathrm{MHz}, \mathrm{CDCl}_{3}\right) \delta-0.05(\mathrm{~s}$, $3 \mathrm{H}),-0.02(\mathrm{~s}, 3 \mathrm{H}), 0.10(\mathrm{~s}, 3 \mathrm{H}), 0.15(\mathrm{~s}, 3 \mathrm{H}), 0.78(\mathrm{~s}, 9 \mathrm{H})$, 0.88 (s, 9H), 2.69 (dd, $1 \mathrm{H}, J=10.2,17.5 \mathrm{~Hz}), 2.91$ (dd, $1 \mathrm{H}, J$ $=10.6,17.5 \mathrm{~Hz}), 3.63-3.66(\mathrm{~m}, 1 \mathrm{H}), 4.16-4.18(\mathrm{~m}, 1 \mathrm{H}), 4.38$ (dd, $1 \mathrm{H}, J=10.2,18.6 \mathrm{~Hz}), 5.10-5.21(\mathrm{~m}, 2 \mathrm{H}), 5.14(\mathrm{~s}, 1 \mathrm{H})$, $5.99-6.05(\mathrm{~m}, 1 \mathrm{H}), 7.20-7.31(\mathrm{~m}, 10 \mathrm{H})$; MS (FAB) 552.2 $\left(\mathrm{M}^{+}\right)$.

5a $(\mathrm{P}=\mathrm{MOM}):{ }^{1} \mathrm{H}$ NMR $\left(500 \mathrm{MHz}, \mathrm{CDCl}_{3}\right) \delta 2.95(\mathrm{dd}$, $2 \mathrm{H}, J=10.6,3.7 \mathrm{~Hz}), 3.27(\mathrm{~s}, 3 \mathrm{H}), 3.29(\mathrm{~s}, 3 \mathrm{H}), 3.64(\mathrm{t}, 1 \mathrm{H}$, $J=5.3 \mathrm{~Hz}), 4.50-4.64(\mathrm{~m}, 1 \mathrm{H}), 4.51$ (d, $1 \mathrm{H}, J=6.7 \mathrm{~Hz}), 4.61$ (d, $1 \mathrm{H}, J=6.7 \mathrm{~Hz}), 4.71-4.84(\mathrm{~m}, 1 \mathrm{H}), 4.81(\mathrm{~s}, 2 \mathrm{H}), 5.18(\mathrm{~s}$, $1 \mathrm{H}), 5.28-5.37(\mathrm{~m}, 2 \mathrm{H}), 5.73-5.91(\mathrm{~m}, 1 \mathrm{H}), 7.23-7.41(\mathrm{~m}$, $10 \mathrm{H})$.

5b (P=TBDMS): ${ }^{1} \mathrm{H}$ NMR (500 MHz, $\left.\mathrm{CDCl}_{3}\right) \delta 0.0$ (s, $3 \mathrm{H}), 0.04(\mathrm{~s}, 3 \mathrm{H}), 0.09(\mathrm{~s}, 6 \mathrm{H}), 0.87(\mathrm{~s}, 9 \mathrm{H}), 0.89(\mathrm{~s}, 9 \mathrm{H})$, 2.73 (dd, $1 \mathrm{H}, J=10.1,16.9 \mathrm{~Hz}), 2.79$ (dd, $1 \mathrm{H}, J=10.1,16.9$ $\mathrm{Hz}), 3.65-3.70(\mathrm{~m}, 1 \mathrm{H}), 4.05-4.11(\mathrm{~m}, 1 \mathrm{H}), 4.38$ (ddd, $1 \mathrm{H}, J$ $=7.3,10.1,10.1 \mathrm{~Hz}), 5.02-5.14(\mathrm{~m}, 2 \mathrm{H}), 5.14(\mathrm{~s}, 1 \mathrm{H}), 5.74-$ $5.87(\mathrm{~m}, 1 \mathrm{H}), 7.21-7.39(\mathrm{~m}, 10 \mathrm{H})$.

Typical procedure of iodoetheration (Entry 1 in Scheme 2): To a solution of 3-diphenylmethyl-5-[1,2-di(t-butyldimethylsilyloxy)-3-butenyl]isoxazoline, 5a ( $\mathrm{P}=\mathrm{TBDMS}$ ) $(552 \mathrm{mg}, 1.0 \mathrm{mmol})$ in dichloromethane $(15 \mathrm{~mL})$ was slowly added iodine monochloride $(2.0 \mathrm{M}$ in dichloromethane 0.65 $\mathrm{mL}, 1.3 \mathrm{mmol}$ ) at $0^{\circ} \mathrm{C}$ and stirred for $3 \mathrm{~h}$ at $\mathrm{rt}$. The reaction mixture was washed with aqueous $20 \%$ sodium bisulfite solution $(10 \mathrm{~mL} \times 2)$, dried over $\mathrm{MgSO}_{4}$, and concentrated to give an oil. The oily residue was purified by flash column chromatography ( $n$-hexane/ethyl acetate, 5/1) to afford trans-6a (P=TBDMS) (353 $\mathrm{mg}$ ) as pale yellow oils.

trans-6a (P=TBDMS): ${ }^{1} \mathrm{H}$ NMR $\left(500 \mathrm{MHz}, \mathrm{CDCl}_{3}\right) \delta$ $0.17(\mathrm{~s}, 9 \mathrm{H}), 0.20(\mathrm{~s}, 3 \mathrm{H}), 0.75(\mathrm{~s}, 9 \mathrm{H}), 0.77(\mathrm{~s}, 9 \mathrm{H}), 2.55$ (dd, $1 \mathrm{H}, J=8.0,16.2 \mathrm{~Hz}$ ), 2.62 (dd, $1 \mathrm{H}, J=6.2,16.2 \mathrm{~Hz}$ ), 3.20 (d, 2H, $J=10.4 \mathrm{~Hz}), 4.03-4.06(\mathrm{~m}, 1 \mathrm{H}), 4.11-4.13(\mathrm{~m}$, $1 \mathrm{H}), 4.34-4.43(\mathrm{~m}, 2 \mathrm{H}) ;{ }^{13} \mathrm{C} \mathrm{NMR}\left(\mathrm{CDCl}_{3}, 125 \mathrm{MHz}\right) \delta$ $-4.6,-4.4,-4.1,0.9,18.0,18.3,25.6,25.7,77.2,77.7,77.9$, $78.4,82.6,87.7,117.2$; IR (neat) $2952,2922,2858,2253$, $1471,1259,1116,1065 \mathrm{~cm}^{-1}$; MS $m / z 512\left(\mathrm{M}^{+}\right), 455,398$, 241,171 .

trans-6a (P=MOM): ${ }^{1} \mathrm{H}$ NMR $\left(500 \mathrm{MHz}, \mathrm{CDCl}_{3}\right) \delta 2.65$ $(\mathrm{d}, 2 \mathrm{H}, J=11.8 \mathrm{~Hz}), 3.16-3.29(\mathrm{~m}, 2 \mathrm{H}), 3.40(\mathrm{~s}, 3 \mathrm{H}), 3.41(\mathrm{~s}$,
$3 \mathrm{H}), 4.20-4.22(\mathrm{~m}, 1 \mathrm{H}), 4.26-4.28(\mathrm{~m}, 1 \mathrm{H}), 4.38-4.50(\mathrm{~m}$, $2 \mathrm{H}), 4.71(\mathrm{dd}, 2 \mathrm{H}, J=11.4,7.6 \mathrm{~Hz}), 4.74(\mathrm{dd}, 2 \mathrm{H}, J=11.5$, $4.3 \mathrm{~Hz}) ;{ }^{13} \mathrm{C}$ NMR $\left(\mathrm{CDCl}_{3}, 125 \mathrm{MHz}\right) \delta 18.3,56.3,56.4$, $76.8,77.1,80.5,80.8,81.6,96.8,97.4,117.1$; IR (neat) $2922,2851,2249,1466,1151,1021 \mathrm{~cm}^{-1}$; MS $m / z 371\left(\mathrm{M}^{+}\right)$, $310,248,214,198,182$.

cis-6a (P=MOM): 'H NMR $\left(500 \mathrm{MHz}, \mathrm{CDCl}_{3}\right) \delta 2.67(\mathrm{~d}$, $2 \mathrm{H}, J=7.0 \mathrm{~Hz}), 3.22-3.33(\mathrm{~m}, 2 \mathrm{H}), 3.44(\mathrm{~s}, 6 \mathrm{H}), 4.22-4.25$ $(\mathrm{m}, 1 \mathrm{H}), 4.28-4.31(\mathrm{~m}, 1 \mathrm{H}), 4.40-4.52(\mathrm{~m}, 2 \mathrm{H}), 4.70-4.80$ $(\mathrm{m}, 4 \mathrm{H}) ;{ }^{13} \mathrm{C} \mathrm{NMR}\left(\mathrm{CDCl}_{3}, 125 \mathrm{MHz}\right) \delta 18.3,56.3,56.4$, $76.8,77.2,80.5,80.8,81.6,96.8,97.4,117.1$; IR (neat) $2926,2852,2249,1465,1150,1022 \mathrm{~cm}^{-1}$; MS $m / z 371\left(\mathrm{M}^{+}\right)$, $310,248,198,182$.

trans-6b (P=TBDMS): 'H NMR (500 MHz, $\left.\mathrm{CDCl}_{3}\right) \delta$ $0.12(\mathrm{~s}, 3 \mathrm{H}), 1.14(\mathrm{~s}, 6 \mathrm{H}), 0.15(\mathrm{~s}, 3 \mathrm{H}), 0.91(\mathrm{~s}, 9 \mathrm{H}), 0.93(\mathrm{~s}$, $9 \mathrm{H}), 2.68$ (dd, $1 \mathrm{H}, J=17.3,4.8 \mathrm{~Hz}$ ), 2.84 (dd, $1 \mathrm{H}, J=17.3$, $7.6 \mathrm{~Hz}$ ), 3.22 (d, 2H, $J=5.2 \mathrm{~Hz}), 3.93-4.04(\mathrm{~m}, 2 \mathrm{H}), 4.28-$ $4.34(\mathrm{~m}, 2 \mathrm{H}) ;{ }^{13} \mathrm{C} \mathrm{NMR}\left(\mathrm{CDCl}_{3}, 125 \mathrm{MHz}\right) \delta-4.8,-4.4$, $-4.3,-4.2,7.5,18.0,18.3,19.8,25.9,26.0,72.9,76.5,76.9$, $83.4,118.3$; IR (neat) $2929,2857,2250,1471,1253,1161$, $1051 \mathrm{~cm}^{-1}$; MS $m / z 512\left(\mathrm{M}^{+}\right), 454,398,270,241,171$.

cis-6b (P=TBDMS): ${ }^{1} \mathrm{H}$ NMR $\left(500 \mathrm{MHz}, \mathrm{CDCl}_{3}\right) \delta 0.15$ (s, 12H), $0.94(\mathrm{~s}, 18 \mathrm{H}), 2.70-2.76(\mathrm{~m}, 2 \mathrm{H}), 3.28$ (d, 2H, $J=$ $6.0 \mathrm{~Hz}), 4.17-4.23(\mathrm{~m}, 2 \mathrm{H}), 4.26-4.29(\mathrm{~m}, 2 \mathrm{H}) ;{ }^{13} \mathrm{C}$ NMR $\left(\mathrm{CDCl}_{3}, 125 \mathrm{MHz}\right) \delta-4.8,-4.6,-4.5,-4.1,3.6,18.4,18.5$, $20.8,26.1,26.2,73.9,74.3,76.0,81.9,118.3$; IR (neat) $2929,2858,2250,1471,1254,1164,1054 \mathrm{~cm}^{-1}$; HRMS caled for $\mathrm{C}_{19} \mathrm{H}_{38} \mathrm{NO}_{3} \mathrm{Si}_{2} \mathrm{I} 511.143503$, found 511.141785 .

\section{References}

I. (a) Beebe, X.; Schore, N. E.; Kurth, M. J. J. Org. Chem, 1995, 60, 4196. (b) Beebe, X.; Chiappari, C. L.; Kurth, M. J.; Schore, N. E. J. Org. Chem. 1993, 58, 7320. (c) Kurth, M. J.; Rodriguez, M. J.; Olmstead, M. M. J. Org. Chen. 1990, 55, 283. (d) Kurth, M. J.; Rodriguez, M. J. Tetrahedron 1998, 45, 6963.

2. (a) Kim, J. N.; Kim, H. R.; Ryu, E. K. Symth. Commm 1993, 23, 1673. (b) Kanemasa, S.; Nishiuchi, M.; Kamimura, A.; Hori, K.J. Am. Chem. Soc. 1994, 116, 2324. (c) Kanemasa, S.; Kobayashi, S.; Nishiuchi, M.; Yamamoto, H.; Wada, E. Tetrahedron Lett. 1991, 32, 6367. (d) Kanemasa, S.; Nishiuchi, M.; Wada, E. Tetrahedron Lett. 1992, 33, 1357.

3. Kim, H. C.; Woo, S. W.; Seo, M. J.; Jeon, D. J.; No, Z; Kim, H. R. Synlett 2002, 1691 .

4. Kim, H. C.; Seo, M. J.; Kim, J. K.; Lee, J.-D.; No, Z; Kim, H. R. Bull. Korean Chem. Soc. 2004, 25, 133.

5. Crombez-Robert, C.; Benazza, M.; Frechou, C; Demailly, G. Carbohtdr. Res. 1997.303.359.

6. Paquette, L. A.; Bailey, S. J. Org. Chem, $1995,60,7849$.

7. Son, B. S.; Song, B. G; Kim, J. K.; Seo, M. J.; No, Z; Kim, H. R. Heterocycles 2005, 65, 1289 . 